\title{
Investigating the role of magnetic fields in star formation using molecular line profiles
}

\author{
Charles Yin ${ }^{\circledR},{ }^{1 \star}$ Felix D. Priestley ${ }^{1}$ and James Wurster ${ }^{\circledR 2}$ \\ ${ }^{1}$ School of Physics and Astronomy, Cardiff University, Queen's Buildings, The Parade, Cardiff CF24 3AA, UK \\ ${ }^{2}$ SUPA, School of Physics and Astronomy, University of St Andrews, North Haugh, St Andrews, Fife KY16 9SS, UK
}

Accepted 2021 April 6. Received 2021 March 19; in original form 2021 January 18

\begin{abstract}
Determining the importance of magnetic fields in star-forming environments is hampered by the difficulty of accurately measuring both field strength and gas properties in molecular clouds. We post-process three-dimensional non-ideal magnetohydrodynamic simulations of pre-stellar cores with a time-dependent chemical network, and use radiative transfer modelling to calculate self-consistent molecular line profiles. Varying the initial mass-to-flux ratio from subcritical to supercritical results in significant changes to both the intensity and shape of several observationally important molecular lines. We identify the peak intensity ratio of $\mathrm{N}_{2} \mathrm{H}^{+}$to CS lines, and the CS $J=2-1$ blue-to-red peak intensity ratio, as promising diagnostics of the initial mass-to-flux ratio, with $\mathrm{N}_{2} \mathrm{H}^{+} / \mathrm{CS}$ values of $>0.6(<0.2)$ and CS blue/red values of $<3(>5)$ indicating subcritical (supercritical) collapse. These criteria suggest that, despite presently being magnetically supercritical, L1498 formed from subcritical initial conditions.
\end{abstract}

Key words: astrochemistry-MHD-stars: formation-ISM: magnetic fields-ISM: molecules.

\section{INTRODUCTION}

The importance of magnetic fields in the collapse of pre-stellar cores to protostars is still poorly understood. Broadly speaking, theories of star formation can be separated into those that assume prestellar cores form above the critical mass for gravitational collapse (Mouschovias 1976), and those that predict that cores are initially subcritical, and contract until the reduction in magnetic flux due to ambipolar diffusion allows the central region to collapse (Fiedler \& Mouschovias 1993). In the former case, star formation occurs on the free-fall time-scale, $\sim 10^{6} \mathrm{yr}$ under typical molecular cloud conditions, whereas in the latter the relevant time-scale is that of ambipolar diffusion, around an order of magnitude larger (Tassis \& Mouschovias 2004; Banerji et al. 2009). While there are other effects that have an impact on the collapse time-scale, such as the cosmic ray ionization rate, these effects are typically less significant than that of the initial mass-to-flux ratio (Wurster, Bate \& Price 2018).

Collapse time-scales not only affect the rate of conversion of interstellar material into stars, but also change the chemical composition of the infalling gas and ice mantles (Tassis et al. 2012a; Priestley, Viti \& Williams 2018), which is inherited, at least in part, by the subsequent protoplanetary disc (Coutens, Commerçon \& Wakelam 2020; Oberg \& Bergin 2020). The mechanism of pre-stellar collapse can thus have consequences on both smaller and larger scales than of the cores themselves.

Direct measurements of the magnetic field strength in molecular clouds typically find values that favour supercritical models of star formation (Crutcher, Hakobian \& Troland 2009; Crutcher 2012), although some exceptions exist (e.g. Soam et al. 2019). However,

^E-mail: charlesyin42@gmail.com values for the mass-to-flux ratio for pre-stellar objects are often close to the critical value and have large uncertainties (Soam et al. 2018; Beltrán et al. 2019). Concerns have also been raised about the reliability of magnetic field strengths measured via the Zeeman effect and their relation to the gas density, due to assumptions about the abundance of the $\mathrm{OH}$ molecule (Tassis et al. 2014) and the statistical methods employed (Tritsis et al. 2015; Jiang, Li \& Fan 2020).

This has led to alternative tests being proposed, typically exploiting the effect of the increased time-scale in initially subcritical models on the molecular abundances, which can be determined relatively easily from observations. Lippok et al. (2013) used the CO depletion in a sample of starless cores to measure chemical ages $\lesssim 1 \mathrm{Myr}$, suggesting supercritical collapse, while Pagani et al. (2013) reached a similar conclusion for L183 based on deuteration measurements of $\mathrm{N}_{2} \mathrm{H}^{+}$. Lin et al. (2020) found that deuteration profiles imply an age $>1$ Myr for L1512, which is more typical of ambipolar diffusion models. Tassis et al. (2012a) used coupled hydrodynamical-chemical models to identify various molecular abundance ratios that are sensitive to the collapse time-scale, although these models are based on the one-dimensional thin disc approximation.

In Priestley, Wurster \& Viti (2019), we post-processed a fully three-dimensional non-ideal magnetohydrodynamical (MHD) model with a time-dependent chemical network in order to determine the molecular structure of initially subcritical and supercritical collapse models. While several molecules differ by orders of magnitude in abundance in the central regions of the pre-stellar cores, due to enhanced freeze-out in the subcritical models, we found the molecular column density profiles were too similar to distinguish the two cases, due to intervening material along the line of sight. In this paper, we instead focus on the synthetic line profiles of several commonly observed species, taking advantage of the velocity structure to probe regions where subcritical and supercritical models of collapse are clearly distinct. 
Table 1. Initial radius, mass density, hydrogen nucleus density, magnetic field strength, duration, and massto-flux ratios for the LOW-SUP and LOW-SUB non-ideal MHD models. $\lambda$ is the mass-to-flux ratio.

\begin{tabular}{lcccccc}
\hline Model & $R / \mathrm{pc}$ & $\rho / \mathrm{g} \mathrm{cm}^{-3}$ & $n_{\mathrm{H}} / \mathrm{cm}^{-3}$ & $B_{z} / \mu \mathrm{G}$ & $t_{\text {end }} / \mathrm{Myr}$ & $\lambda / \lambda_{\text {crit }}$ \\
\hline LOW-SUP & 0.13 & $3.68 \times 10^{-20}$ & $1.57 \times 10^{4}$ & 9.1 & 0.347 & 5 \\
LOW-SUB & 0.13 & $3.68 \times 10^{-20}$ & $1.57 \times 10^{4}$ & 91 & 0.972 & 0.5 \\
\hline
\end{tabular}
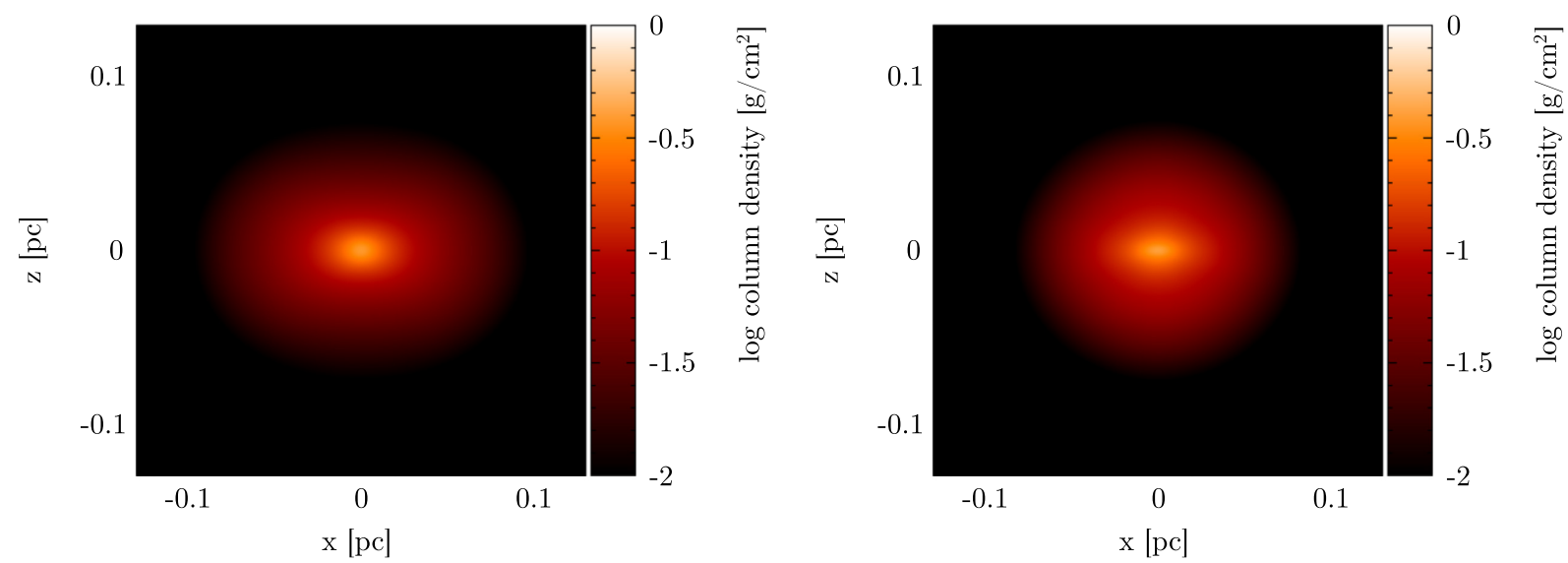

Figure 1. Column density of the LOW-SUB (left) and LOW-SUP (right) models, viewed side-on.

\section{METHOD}

Following Priestley et al. (2019), we use the PHANTOM smoothed particle (magneto)hydrodynamics (SPH) code (Price et al. 2018) to run models of spherical, static, uniform density pre-stellar cores with a constant magnetic field in the $z$-direction. The ambipolar diffusion coefficient is calculated using the NICIL library (Wurster 2016); as NICIL does not include molecular ions, which are the dominant ionized species at the densities we investigate, we assume that the ion density is given by

$n_{\mathrm{i}} / n_{\mathrm{H}}=10^{-7}\left(\frac{n_{\mathrm{H}}}{10^{3} \mathrm{~cm}^{-3}}\right)^{-0.6}$,

which accurately reproduces the ion-neutral relation produced by full, time-dependent chemical networks to within a factor of a few over the relevant range of densities (Tassis et al. 2012b; Priestley et al. 2019).

We assume an isothermal equation of state with a sound speed $\left(c_{\mathrm{s}}\right)$ of $0.2 \mathrm{~km} \mathrm{~s}^{-1}$, corresponding to molecular gas at $\sim 10 \mathrm{~K}$. The cores are surrounded by a background medium with the density reduced by a factor of 30 , with the temperature increased by an equivalent factor to ensure pressure balance. ${ }^{1}$ As the high-density models in Priestley et al. (2019) resulted in anomalously low CO abundances compared to observed pre-stellar cores, we focus on the low-density case, with a core mass $(M)$ of $5 \mathrm{M}_{\odot}$ and a radius $(R)$ of $0.13 \mathrm{pc}$, giving an initial hydrogen nucleus density $\left(n_{\mathrm{H}}\right)$ of $1.57 \times 10^{4} \mathrm{~cm}^{-3}$. We consider models with mass-to-flux ratios of 5 (LOW-SUP) and 0.5 (LOW-SUB) times the critical value (Mouschovias 1976), equivalent to initial magnetic field strengths $B_{z}=9.1$ and $91 \mu \mathrm{G}^{2}{ }^{2}$ The models

\footnotetext{
${ }^{1}$ The models in Priestley et al. (2019) were erroneously run with a background medium temperature of $10 \mathrm{~K}$, affecting the core dynamics and subsequent chemical evolution. The authors of that paper have submitted an erratum; the results presented here have the correct background temperature.

${ }^{2}$ These are slightly higher than those in Priestley et al. (2019) due to a minor change to the definition of the critical mass-to-flux ratio.
}

are terminated when the central density reaches $n_{\mathrm{H}}=2 \times 10^{6} \mathrm{~cm}^{-3}$ which occurs after 0.305 and $0.964 \mathrm{Myr}$ for the LOW-SUP and LOW-SUB models, respectively. The models are run with $\sim 200000$ particles, giving a particle mass of $3.5 \times 10^{-5} \mathrm{M}_{\odot}$. Model properties are listed in Table 1. The final column density structures for both models, viewed side-on, are shown in Fig. 1.

We post-process a randomly selected subset of 10000 particles with the UCLCHEM chemical evolution code (Holdship et al. 2017) to obtain the three-dimensional molecular structure of each model core, using the UMIST12 reaction rate network (McElroy et al. 2013) and the low-metal elemental abundances from Lee et al. (1998). We assume a constant gas/grain temperature $(10 \mathrm{~K})$ and cosmic ray ionization rate $\left(1.3 \times 10^{-17} \mathrm{~s}^{-1}\right)$, and set the radiation field to zero; pre-stellar cores are typically located within larger molecular clouds, and as such are shielded from any external radiation field.

We use LIME (Brinch \& Hogerheijde 2010) to calculate line intensities from our MHD-chemical modelling results for a number of observationally important molecules, taking molecular data from the LAMDA data base (Schöier et al. 2005). Density, molecular abundance, and other properties are assigned to sampling points from the nearest neighbour SPH particle that was post-processed chemically. We use 10000 spatial grid points and 201 velocity channels with a spacing of $0.01 \mathrm{~km} \mathrm{~s}^{-1}$, which we have confirmed are sufficient that our results are converged. We assume isotopic ratios of ${ }^{12} \mathrm{C} /{ }^{13} \mathrm{C}=100$ and ${ }^{16} \mathrm{O} /{ }^{18} \mathrm{O}=500$. We evaluate line profiles at the simulation end points (when the central density reaches $n_{\mathrm{H}}=2 \times 10^{6} \mathrm{~cm}^{-3}$ ) by averaging the line intensity over all pixels within a radius of $0.13 \mathrm{pc}$ of the centre of the image.

\section{RESULTS}

Figs 2 and 3 show the average line profiles for a number of observationally relevant molecules, for side-on ( $x-z$ plane $)$ and faceon ( $x-y$ plane) orientations with respect to the magnetic field axis, respectively. To better compare the shapes rather than the absolute 

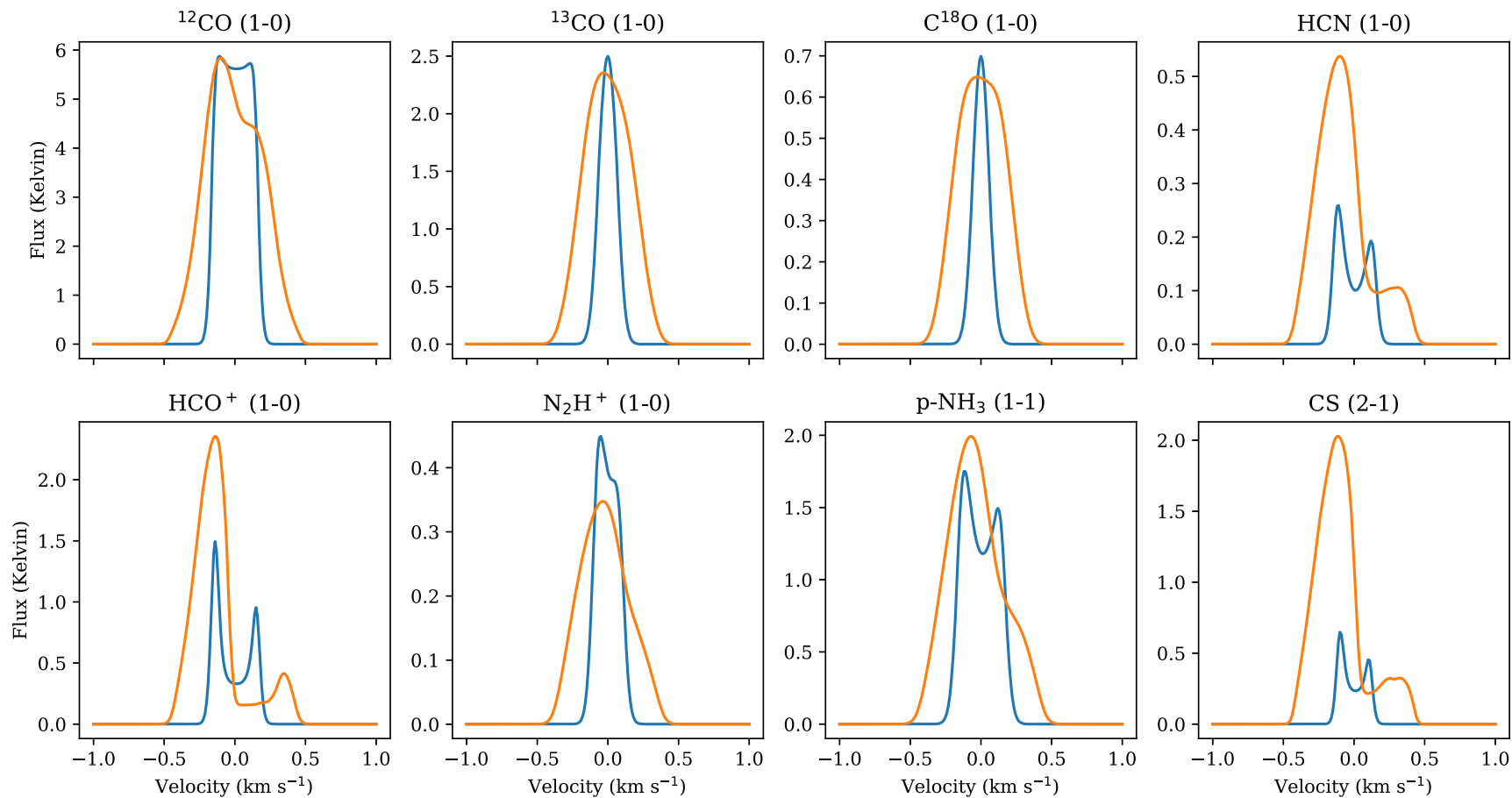

Figure 2. Line profiles for various species viewed side-on. Each species is labelled above the plot. The blue line is the LOW-SUB model, while the orange line is the LOW-SUP model.
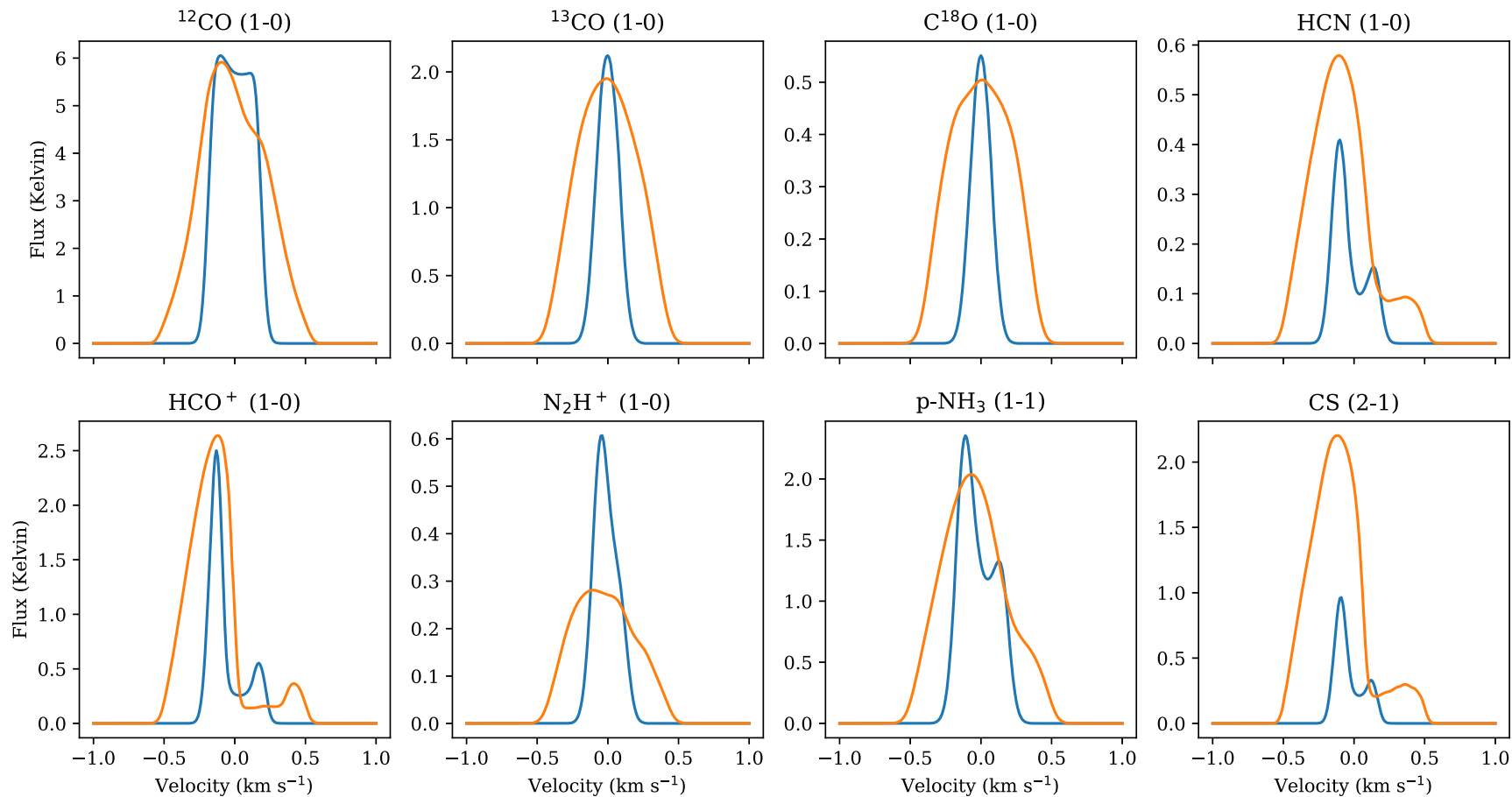

Figure 3. Line profiles for various species viewed face-on. Each species is labelled above the plot. The blue line is the LOW-SUB model, while the orange line is the LOW-SUP model.

intensities, Figs 4 and 5 show the line profiles normalized to the peak intensity. Depending on whether the line appears singly or doubly peaked, the profiles were fitted with either a single or double Gaussian profile, the fit parameters of which are given in Tables 2 and 3. While the profiles are not necessarily Gaussian, this provides a simple way of comparing displacements and line widths.

All three CO isotopologues have similar peak intensities for the subcritical and supercritical models, but the LOW-SUP line profiles are much broader, due to less depletion in the central, high-velocity 

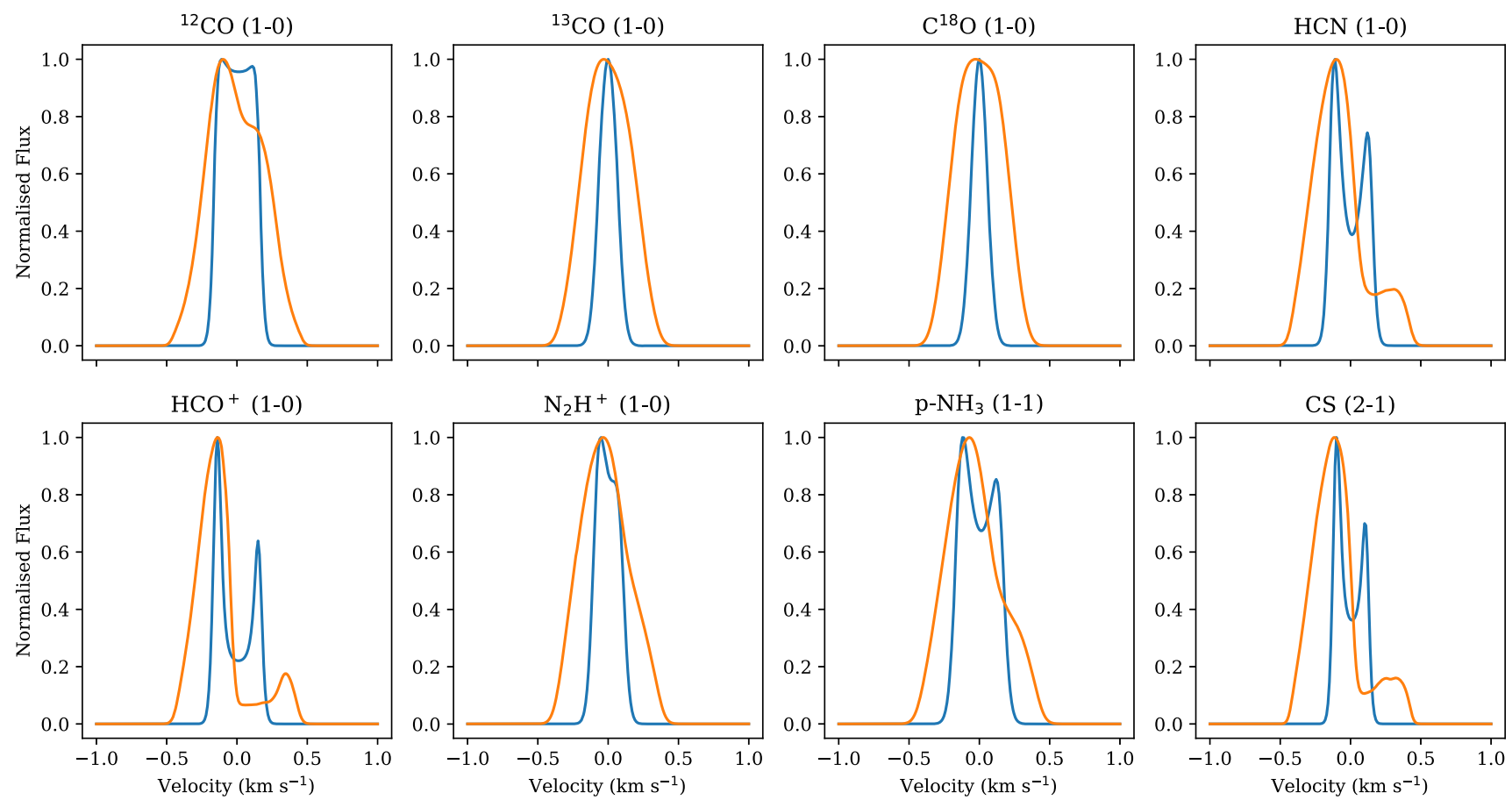

Figure 4. Normalized line profiles for various species viewed side-on. Each species is labelled above the plot. The blue line is the LOW-SUB model, while the orange line is the LOW-SUP model.
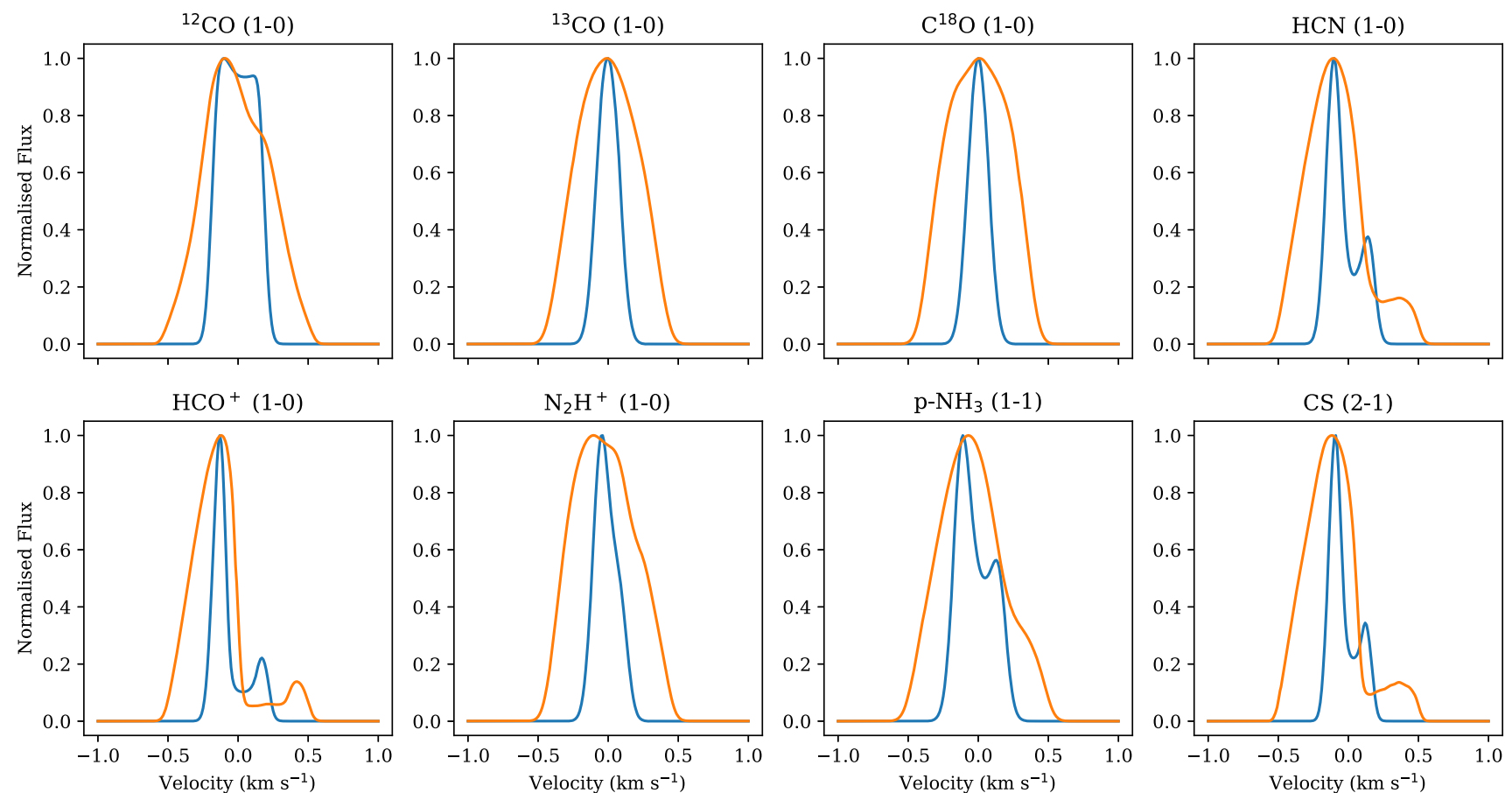

Figure 5. Normalized line profiles for various species viewed face-on. Each species is labelled above the plot. The blue line is the LOW-SUB model, while the orange line is the LOW-SUP model.

regions, as can be seen in Fig. 6. HCN and CS are both significantly weaker for LOW-SUB due to lower abundances throughout the core (CS is shown in Fig. 6), whereas the p- $\mathrm{NH}_{3}$ and $\mathrm{N}_{2} \mathrm{H}^{+}$lines are of comparable or even greater strength in the LOW-SUB model as they are far less affected by depletion. $\mathrm{HCO}^{+}$shows the greatest variation with viewing angle; the mid-plane abundance (shown in Fig. 7) is lower, but the $z$-axis abundance higher, for the LOW-SUB model, resulting in the contrasting behaviour.

The asymmetric double-peaked appearance of some lines, with the blue peak stronger than the red, is due to self-absorption by 
Table 2. Parameters for one or two Gaussian curves fitted to the line profile of various species when viewed side-on. For each species, the first row is the LOW-SUB model, while the second row is the LOW-SUP model.

\begin{tabular}{|c|c|c|c|c|c|c|}
\hline & \multicolumn{3}{|c|}{ Gaussian 1} & \multicolumn{3}{|c|}{ Gaussian 2} \\
\hline & $\begin{array}{l}\text { Height } \\
(\mathrm{K})\end{array}$ & $\begin{array}{l}\text { Centre } \\
\left(\mathrm{m} \mathrm{s}^{-1}\right)\end{array}$ & $\begin{array}{l}\text { Width } \\
\left(\mathrm{m} \mathrm{s}^{-1}\right)\end{array}$ & $\begin{array}{l}\text { Height } \\
(\mathrm{K})\end{array}$ & $\begin{array}{l}\text { Centre } \\
\left(\mathrm{m} \mathrm{s}^{-1}\right)\end{array}$ & $\begin{array}{l}\text { Width } \\
\left(\mathrm{m} \mathrm{s}^{-1}\right)\end{array}$ \\
\hline \multirow[t]{2}{*}{${ }^{12} \mathrm{CO}$} & 6.10 & -87.5 & 63.5 & 6.07 & 83.4 & 66.3 \\
\hline & 5.67 & -108 & 134 & 3.59 & 181 & 112 \\
\hline \multirow[t]{2}{*}{${ }^{13} \mathrm{CO}$} & 2.55 & -0.270 & 61.3 & - & - & - \\
\hline & 2.48 & -4.13 & 170 & - & - & - \\
\hline \multirow[t]{2}{*}{$\mathrm{C}^{18} \mathrm{O}$} & 0.702 & -0.0533 & 55.6 & - & - & - \\
\hline & 0.707 & 0.213 & 173 & - & - & - \\
\hline \multirow[t]{2}{*}{$\mathrm{HCN}$} & 0.248 & -102 & 489 & 0.179 & 97.8 & 59.1 \\
\hline & 0.547 & -125 & 138 & 0.111 & 303 & 81.7 \\
\hline \multirow[t]{2}{*}{$\mathrm{HCO}^{+}$} & 1.38 & -134 & 36.2 & 0.659 & 115 & 74.0 \\
\hline & 2.38 & -178 & 104 & 0.369 & 323 & 88.4 \\
\hline \multirow[t]{2}{*}{$\mathrm{N}_{2} \mathrm{H}^{+}$} & 0.430 & -57.5 & 466 & 0.362 & 57.5 & 49.1 \\
\hline & 0.355 & -48.5 & 161 & 0.0587 & 251 & 78.5 \\
\hline \multirow[t]{2}{*}{$\mathrm{p}-\mathrm{NH}_{3}$} & 1.74 & -101 & 63.7 & 1.50 & 97.6 & 69.7 \\
\hline & 2.00 & -80.7 & 164 & 0.493 & 284 & 90.4 \\
\hline \multirow[t]{2}{*}{ CS } & 0.609 & -89.3 & 361 & 0.387 & 78.0 & 54.3 \\
\hline & 2.07 & -141 & 123 & 0.365 & 297 & 83.2 \\
\hline
\end{tabular}

Table 3. Parameters for one or two Gaussian curves fitted to the line profile of various species when viewed from the $z$-axis. For each species, the first row is the LOW-SUB model, while the second row is the LOW-SUP model.

\begin{tabular}{|c|c|c|c|c|c|c|}
\hline & \multicolumn{3}{|c|}{ Gaussian 1} & \multicolumn{3}{|c|}{ Gaussian 2} \\
\hline & $\begin{array}{l}\text { Height } \\
\text { (K) }\end{array}$ & $\begin{array}{l}\text { Centre } \\
\left(\mathrm{m} \mathrm{s}^{-1}\right)\end{array}$ & $\begin{array}{l}\text { Width } \\
\left(\mathrm{m} \mathrm{s}^{-1}\right)\end{array}$ & $\begin{array}{l}\text { Height } \\
(\mathrm{K})\end{array}$ & $\begin{array}{l}\text { Centre } \\
\left(\mathrm{m} \mathrm{s}^{-1}\right)\end{array}$ & $\begin{array}{r}\text { Width } \\
\left(\mathrm{m} \mathrm{s}^{-1}\right)\end{array}$ \\
\hline \multirow[t]{2}{*}{${ }^{12} \mathrm{CO}$} & 6.23 & -95.1 & 75.3 & 5.87 & 97.5 & 74.3 \\
\hline & 5.74 & -109 & 168 & 3.02 & 223 & 136 \\
\hline \multirow[t]{2}{*}{${ }^{13} \mathrm{CO}$} & 2.18 & -1.33 & 76.4 & - & - & - \\
\hline & 2.05 & -4.79 & 222 & - & - & - \\
\hline \multirow[t]{2}{*}{$\mathrm{C}^{18} \mathrm{O}$} & 0.558 & -0.450 & 71.6 & - & - & - \\
\hline & 0.544 & 1.27 & 231 & - & - & - \\
\hline \multirow[t]{2}{*}{$\mathrm{HCN}$} & 0.407 & -101 & 57.3 & 0.151 & 120 & 65.1 \\
\hline & 0.594 & -127 & 178 & 0.0903 & 391 & 780 \\
\hline \multirow[t]{2}{*}{$\mathrm{HCO}^{+}$} & 2.47 & -133 & 46.0 & 0.474 & 139 & 79.5 \\
\hline & 2.68 & -176 & 137 & 0.340 & 404 & 84.1 \\
\hline \multirow{2}{*}{$\mathrm{N}_{2} \mathrm{H}^{+}$} & 0.589 & -49.4 & 60.0 & 0.256 & 79.3 & 54.9 \\
\hline & 0.244 & 60.5 & 197 & 0.167 & -218 & 122 \\
\hline \multirow[t]{2}{*}{$\mathrm{p}-\mathrm{NH}_{3}$} & 2.33 & -105 & 68.8 & 1.35 & 110 & 77.5 \\
\hline & 2.07 & -76.1 & 211 & 0.353 & 371 & 86.6 \\
\hline \multirow[t]{2}{*}{ CS } & 0.953 & -93.3 & 47.2 & 0.316 & 103 & 60.4 \\
\hline & 2.28 & -144 & 161 & 0.314 & 380 & 80.9 \\
\hline
\end{tabular}

inflowing material, i.e. an inverse P Cygni effect. This is commonly seen in observations of actual pre-stellar cores (Tafalla et al. 2002, 2006). For comparison, the line-centre optical depth for the LOWSUB model viewed face-on is $\sim 0.5$ for ${ }^{13} \mathrm{CO}$ (single peak) and $\sim 15$ for HCN (double peaked). In both orientations, the LOW-SUP model results in broader lines. These effects are due to the larger abundances throughout the core in the faster evolving supercritical model, where molecules have less time to freeze out on to grain surfaces, as can be seen in Fig. 6 for CO and CS. However, these effects are unlikely to make useful diagnostics for the mechanism of collapse. The differences involved are not large (typically a factor of a few), and are likely to be strongly affected by the initial density and cloud size, along with other effects we do not consider such as rotation. We thus focus on differences in the properties of lines relative to each other, which should be much less sensitive to the initial conditions.

Table 4 lists the ratios of blue to red peaks, as measured by our Gaussian fits. There is a significant difference between the LOWSUB and LOW-SUP models in the ${ }^{12} \mathrm{CO}, \mathrm{HCN}, \mathrm{HCO}^{+}, \mathrm{N}_{2} \mathrm{H}^{+}$, p$\mathrm{NH}_{3}$, and $\mathrm{CS}$ lines when viewed side-on. However, when viewed face-on the difference is not present for $\mathrm{HCO}^{+}$, and is reversed in $\mathrm{N}_{2} \mathrm{H}^{+}$. For these molecules, the abundance profiles face-on axis for the LOW-SUB model reach levels comparable to or larger than those in the LOW-SUP model at small radii, resulting in larger column densities and thus stronger absorption when viewed face-on. This can be seen for $\mathrm{HCO}^{+}$in Fig. 7. As cores may be observed at any inclination, peak ratios for these molecules cannot be used to discriminate between models. $\mathrm{p}-\mathrm{NH}_{3}$ shows blue-to-red peak intensity ratios of $\sim 1.5$ for the LOW-SUP model and $\sim 4-6$ for the LOW-SUB model, but the red 'peak' for the LOW-SUP model is barely detectable as such, appearing as more of a shoulder on the line profile. CS and HCN both have blue/red peak intensity ratios $\lesssim 3$ for the LOW-SUB model, and $\gtrsim 5$ for LOW-SUP, regardless of inclination, and are thus more promising as tracers of magnetic criticality.

In addition to considering the shape of the line, the ratios of peak intensities of the lines produced by different species are also potentially useful. These are given in Tables 5 and 6 for the side-on and face-on cases, respectively. Some differences are consistent between the two models regardless of orientation. These include $\mathrm{N}_{2} \mathrm{H}^{+}$and $\mathrm{HCN} ; \mathrm{N}_{2} \mathrm{H}^{+}$and $\mathrm{HCO}^{+} ;{ }^{12} \mathrm{CO}$ and $\mathrm{CS} ;{ }^{13} \mathrm{CO}$ and $\mathrm{CS} ; \mathrm{C}^{18} \mathrm{O}$ and $\mathrm{CS} ; \mathrm{N}_{2} \mathrm{H}^{+}$and $\mathrm{CS}$; and $\mathrm{N}_{2} \mathrm{H}^{+}$and CS. These are all parings of species with a prominent self-absorption feature with ones without. In particular, we note that the $\mathrm{N}_{2} \mathrm{H}^{+} / \mathrm{CS}$ and $\mathrm{HCN} / \mathrm{N}_{2} \mathrm{H}^{+}$peak intensity ratios are not greatly affected by viewing angle, but they do vary significantly between the LOW-SUB and LOW-SUP models. The LOW-SUB model has $\mathrm{N}_{2} \mathrm{H}^{+} / \mathrm{CS}>0.6$ and $\mathrm{HCN} / \mathrm{N}_{2} \mathrm{H}^{+}<1$, whereas the LOW-SUP model has $\mathrm{N}_{2} \mathrm{H}^{+} / \mathrm{CS}$ $<0.2$ and $\mathrm{HCN} / \mathrm{N}_{2} \mathrm{H}^{+}>1$, due to comparable $\mathrm{N}_{2} \mathrm{H}^{+}$line strengths but significantly weaker $\mathrm{CS} / \mathrm{HCN}$ intensity for the LOW-SUB model.

\section{DISCUSSION}

The synthetic lines presented here are from simulations of static, uniform density spheres, and so are not necessarily representative of real pre-stellar cores. Even restricting ourselves to this model setup, changing the cloud mass or density may alter the line profiles by larger amounts than the differences between subcritical and supercritical models. For this reason, we have focused on diagnostics that should not depend on the absolute line intensity, are likely to be least sensitive to initial cloud parameters, and that show a clear dichotomy between the two models. The blue/red peak ratio of the CS and $\mathrm{HCN}$ lines and the intensity ratios of those molecules with $\mathrm{N}_{2} \mathrm{H}^{+}$are easily interpreted as they are primarily due to the longer duration of freeze-out in initially subcritical cores, resulting in less self-absorption and lower peak intensity for CS and HCN while $\mathrm{N}_{2} \mathrm{H}^{+}$is relatively unaffected. Other effects not considered here, such as the cosmic ray ionization rate (Wurster et al. 2018) and the initial magnetic field strength (Bate, Tricco \& Price 2014), can also change the collapse time-scale. However, these effects do not change the collapse time-scale to the extent of the difference between supercritical and subcritical models (Machida, Higuchi \& Okuzumi 2018). As lower initial densities result in more strongly delayed collapse (the ratio of ionized to neutral species, and thus the ambipolar diffusion time-scales, is larger at lower density), the 

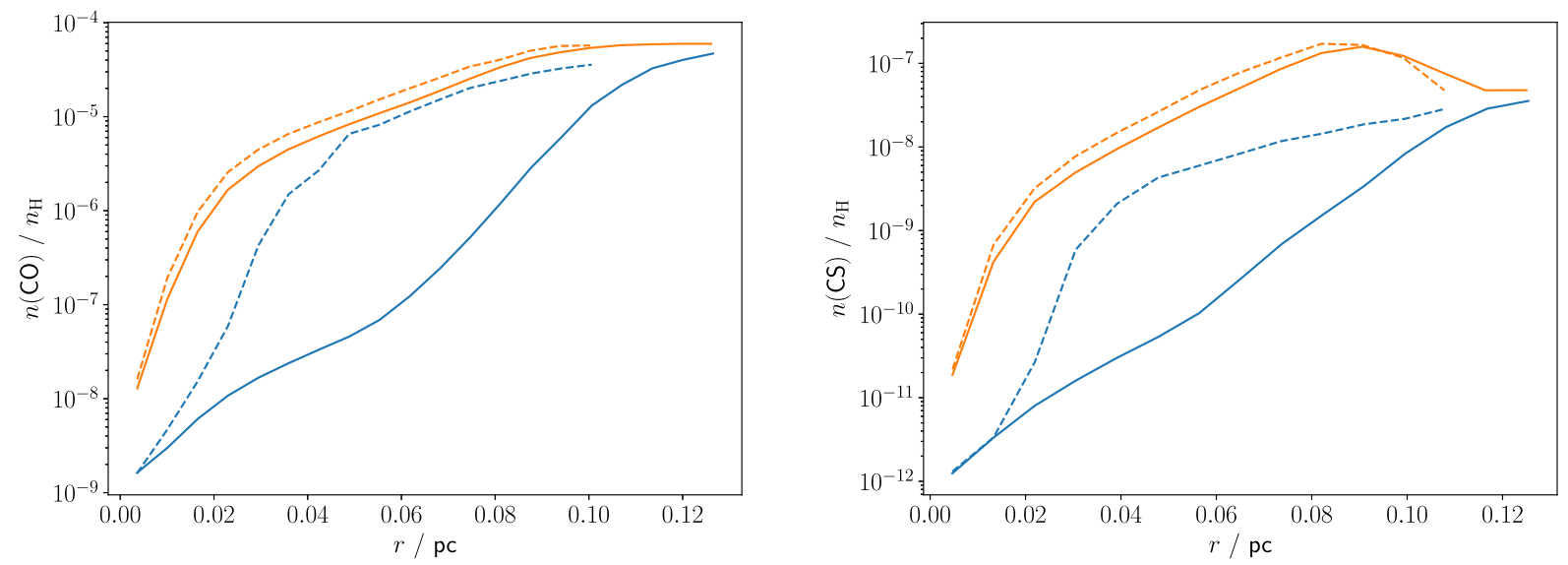

Figure 6. Mid-plane (solid lines) and $z$-axis (dashed lines) CO (left-hand panel) and CS (right-hand panel) abundance profiles for the LOW-SUP (orange) and LOW-SUB (blue) models.

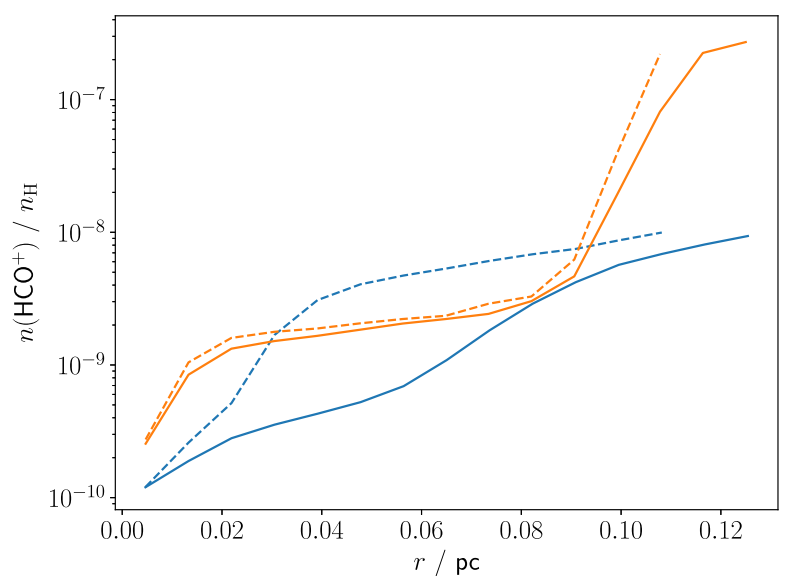

Figure 7. Mid-plane (solid lines) and $z$-axis (dashed lines) $\mathrm{HCO}^{+}$abundance profiles for the LOW-SUP (orange) and LOW-SUB (blue) models.

Table 4. Ratio of the intensity of blue to red peaks. The first line lists the ratio for the LOW-SUB model, and the second line lists the ratio for the LOW-SUP model.

\begin{tabular}{lcc}
\hline Species & Side-on & $z$-direction \\
\hline${ }^{12} \mathrm{CO}$ & 1.00 & 1.06 \\
& 1.58 & 1.90 \\
${ }^{13} \mathrm{CO}$ & - & - \\
& - & - \\
$\mathrm{C}^{18} \mathrm{O}$ & - & - \\
& - & - \\
$\mathrm{HCN}$ & 1.38 & 2.70 \\
& 4.93 & 6.58 \\
$\mathrm{HCO}^{+}$ & 2.09 & 5.21 \\
& 6.45 & 7.88 \\
$\mathrm{~N}_{2} \mathrm{H}^{+}$ & 1.19 & 2.30 \\
& 6.05 & 0.684 \\
$\mathrm{p}-\mathrm{NH}_{3}$ & 1.16 & 1.73 \\
& 4.06 & 5.86 \\
$\mathrm{CS}$ & 1.57 & 3.02 \\
& 5.67 & 7.26 \\
\hline
\end{tabular}

Table 5. Peak intensity ratios of different lines when viewed side-on. For each ratio, the first line is the LOW-SUB model, and the second line is the LOW-SUP model. Each figure is the peak intensity of the species below divided by the species to its left.

\begin{tabular}{|c|c|c|c|c|c|c|c|}
\hline \multirow[t]{2}{*}{${ }^{13} \mathrm{CO}$} & 2.35 & - & - & - & - & - & - \\
\hline & 2.48 & - & - & - & - & - & - \\
\hline \multirow[t]{2}{*}{$\mathrm{C}^{18} \mathrm{O}$} & 8.40 & 3.58 & - & - & - & - & - \\
\hline & 9.00 & 3.63 & - & - & - & - & - \\
\hline \multirow[t]{2}{*}{$\mathrm{HCN}$} & 22.7 & 9.64 & 2.70 & - & - & - & - \\
\hline & 10.9 & 4.38 & 1.21 & - & - & - & - \\
\hline \multirow[t]{2}{*}{$\mathrm{HCO}^{+}$} & 3.93 & 1.67 & 0.468 & 0.173 & - & - & - \\
\hline & 2.48 & 1.00 & 0.275 & 0.228 & - & - & - \\
\hline \multirow[t]{2}{*}{$\mathrm{N}_{2} \mathrm{H}^{+}$} & 13.1 & 5.56 & 1.56 & 0.577 & 3.33 & - & - \\
\hline & 16.8 & 6.78 & 1.87 & 1.55 & 6.78 & - & - \\
\hline \multirow[t]{2}{*}{$\mathrm{p}-\mathrm{NH}_{3}$} & 3.36 & 1.43 & 0.399 & 0.148 & 0.854 & 0.257 & - \\
\hline & 2.93 & 1.18 & 0.325 & 0.270 & 1.18 & 0.174 & - \\
\hline \multirow[t]{3}{*}{ CS } & 9.06 & 3.86 & 1.078 & 0.400 & 2.31 & 0.693 & 2.70 \\
\hline & 2.88 & 1.16 & 0.320 & 0.265 & 1.16 & 0.171 & 0.983 \\
\hline & ${ }^{12} \mathrm{CO}$ & ${ }^{13} \mathrm{CO}$ & $\mathrm{C}^{18} \mathrm{O}$ & $\mathrm{HCN}$ & $\mathrm{HCO}^{+}$ & $\mathrm{N}_{2} \mathrm{H}^{+}$ & $\mathrm{p}-\mathrm{NH}_{3}$ \\
\hline
\end{tabular}

Table 6. Peak intensity ratios of different lines when viewed face-on. For each ratio, the first line is the LOW-SUB model, and the second line is the LOW-SUP model. Each figure is the peak intensity of the species below divided by the species to its left.

\begin{tabular}{|c|c|c|c|c|c|c|c|}
\hline \multirow[t]{2}{*}{${ }^{13} \mathrm{CO}$} & 2.86 & - & - & - & - & - & - \\
\hline & 3.03 & - & - & - & - & - & - \\
\hline \multirow[t]{2}{*}{$\mathrm{C}^{18} \mathrm{O}$} & 11.0 & 3.85 & - & - & - & - & - \\
\hline & 11.7 & 3.87 & - & - & - & - & - \\
\hline \multirow[t]{2}{*}{$\mathrm{HCN}$} & 14.8 & 5.18 & 1.35 & - & - & - & - \\
\hline & 10.2 & 3.37 & 0.871 & - & - & - & - \\
\hline \multirow[t]{2}{*}{$\mathrm{HCO}^{+}$} & 2.42 & 0.848 & 0.220 & 0.164 & - & - & - \\
\hline & 2.24 & 0.740 & 0.191 & 0.219 & - & - & - \\
\hline \multirow[t]{2}{*}{$\mathrm{N}_{2} \mathrm{H}^{+}$} & 9.98 & 3.49 & 0.908 & 0.674 & 4.12 & - & - \\
\hline & 21.0 & 6.94 & 1.79 & 2.059 & 9.39 & - & - \\
\hline \multirow[t]{2}{*}{$\mathrm{p}-\mathrm{NH}_{3}$} & 2.57 & 0.901 & 0.234 & 0.174 & 1.06 & 0.258 & - \\
\hline & 2.90 & 0.958 & 0.248 & 0.284 & 1.30 & 0.138 & - \\
\hline \multirow[t]{3}{*}{ CS } & 6.28 & 2.20 & 0.571 & 0.425 & 2.59 & 0.629 & 2.44 \\
\hline & 2.69 & 0.886 & 0.229 & 0.263 & 1.20 & 0.128 & 0.925 \\
\hline & ${ }^{12} \mathrm{CO}$ & ${ }^{13} \mathrm{CO}$ & $\mathrm{C}^{18} \mathrm{O}$ & $\mathrm{HCN}$ & $\mathrm{HCO}^{+}$ & $\mathrm{N}_{2} \mathrm{H}^{+}$ & $\mathrm{p}-\mathrm{NH}_{3}$ \\
\hline
\end{tabular}



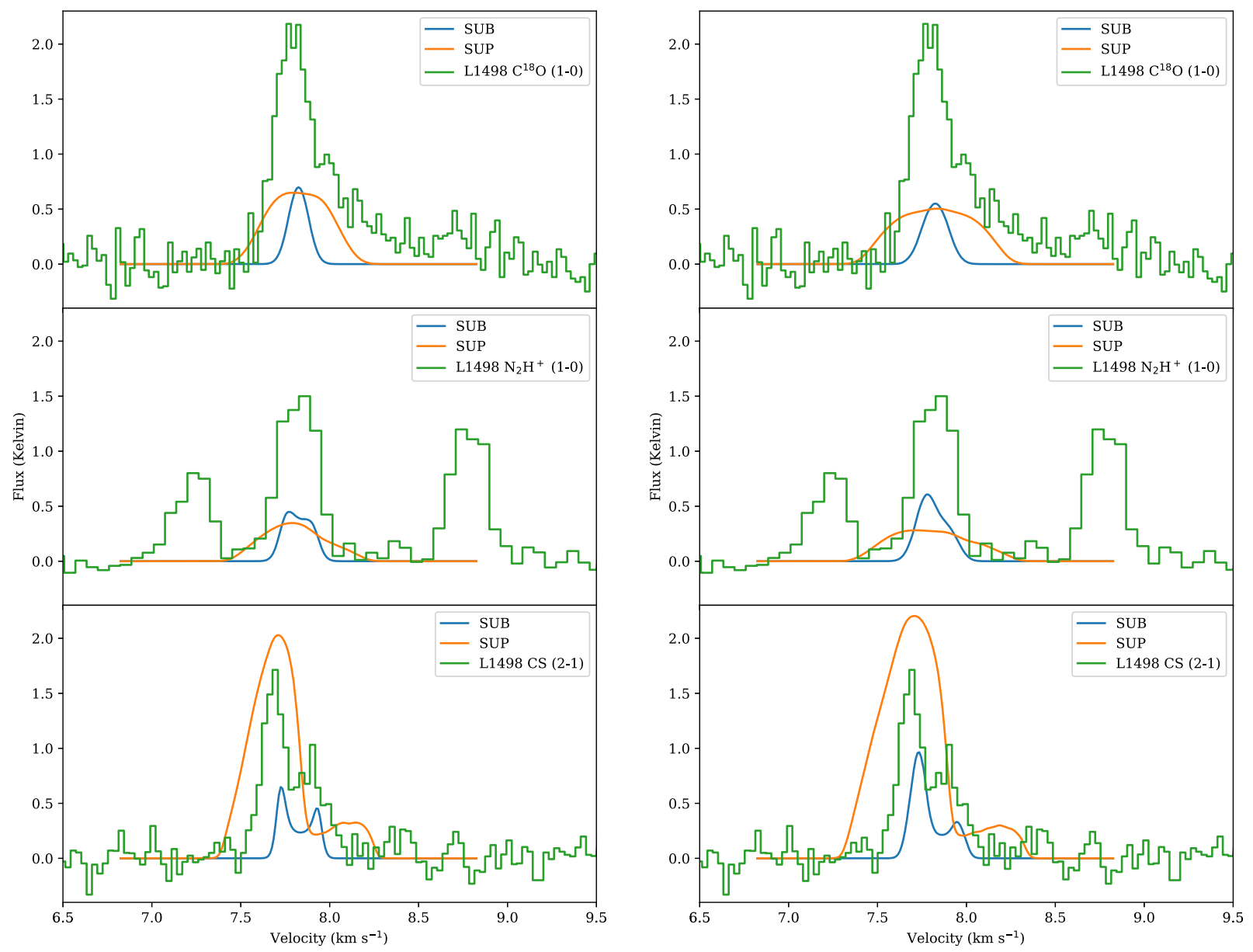

Figure 8. Comparison of the synthetic line profiles and observations of L1498 presented in Tafalla et al. (2002), assuming a face-on (left) or side-on (right) view.

difference in intensity ratio between models discussed here should be even more extreme in this case, while higher initial densities than the $10^{4} \mathrm{~cm}^{-3}$ we assume here seem to be in tension with observed molecular abundances (Priestley et al. 2019). It would, however, be useful to confirm whether these signatures are robust to the model parameters, and we intend to conduct a more detailed study of the parameter space in the near future.

With the above caveats, we can compare our model output to observational data as a test of consistency. Of the five pre-stellar cores studied in Tafalla et al. (2002), only L1498 clearly shows signs of infall in its line profiles, with Kirk, Ward-Thompson \& Crutcher (2006) finding the magnetic flux to be slightly supercritical. Therefore, we choose L1498 as a test case. The synthetic line profiles for $\mathrm{C}^{18} \mathrm{O}$, $\mathrm{N}_{2} \mathrm{H}^{+}$, and CS are overlaid on the observed lines for L1498 in Fig. 8 - we shift the synthetic profile in velocity so that the peak intensity is at the same location as the observed maximum, but otherwise do not modify our data. The observed $\mathrm{C}^{18} \mathrm{O}$ and $\mathrm{N}_{2} \mathrm{H}^{+}$intensities are stronger by a factor of $\sim 2$ than our model line profiles. For CS, the LOW-SUP model has a comparable peak intensity, but a much greater blue/red intensity ratio than observed, whereas the LOW-SUB model is still somewhat weaker than the Tafalla et al. (2002) data but with a similar blue/red ratio. The observed $\mathrm{N}_{2} \mathrm{H}^{+} / \mathrm{CS}$ intensity ratio of $\sim 1$ is also in much better agreement with the LOW-SUB model.
L1498 has an observed size of $\sim 100$ arcsec, corresponding to a physical radius of $\sim 0.06 \mathrm{pc}$ for a distance of $140 \mathrm{pc}$ as typically assumed (Tafalla et al. 2002). If we instead compare the data with model line profiles from the inner $0.06 \mathrm{pc}$ region of our models, shown in Fig. 9, we find that the LOW-SUB model provides a good match to the $\mathrm{N}_{2} \mathrm{H}^{+}$and CS profiles regardless of orientation, whereas the LOW-SUP CS intensity is far greater than that observed. We thus favour a subcritical interpretation for the L1498 data. This is not necessarily in conflict with the supercritical mass-to-flux ratio found by Kirk et al. (2006); their measurement corresponds to the present value of the mass-to-flux ratio in the dense structure identified as a core, whereas the molecular line data are sensitive to the initial value in the material that eventually formed L1498.

The LOW-SUB model is a factor of $\sim 2$ weaker than the observed $\mathrm{C}^{18} \mathrm{O}$ emission even when only the central region is considered. Tafalla et al. (2002) note that there may be contributions to this line from unrelated ambient gas, and the central column density of L1498 ( $\sim 10^{22} \mathrm{~cm}^{-2}$; Kirk et al. 2006$)$ is lower than the LOW-SUB model at the point where we produce line profiles $\left(7.8 \times 10^{22} \mathrm{~cm}^{-2}\right)$, which may suggest that a more appropriate comparison would be with an earlier, less-depleted phase with correspondingly stronger $\mathrm{CO}$ emission. Alternatively, our chosen model parameters may be entirely inappropriate for L1498, and the good agreement between the modelled and observed $\mathrm{N}_{2} \mathrm{H}^{+}$and CS lines is simply a coin- 

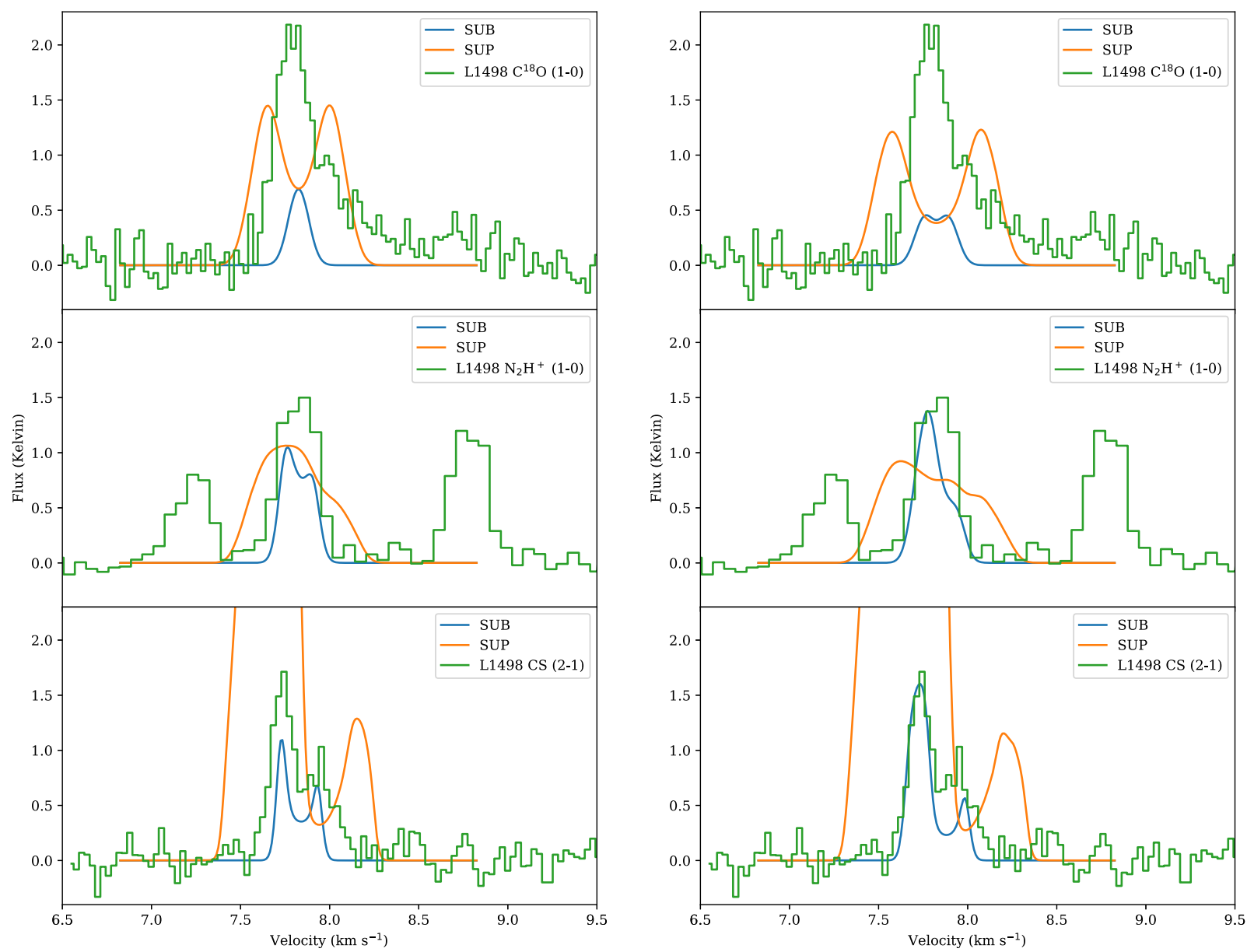

Figure 9. Comparison of the synthetic line profiles and observations of L1498 presented in Tafalla et al. (2002), assuming a face-on (left) or side-on (right) view. Model line profiles are extracted from the central $0.06 \mathrm{pc}$.

cidence. None the less, it is clear from Fig. 9 that the differences between subcritical and supercritical line profiles are large enough to be observationally significant, making this method a promising (and complementary) approach to determining the role of magnetic fields in star formation.

\section{CONCLUSIONS}

We have post-processed non-ideal MHD simulations of collapsing magnetically subcritical/supercritical pre-stellar cores with both a time-dependent chemical network and a line radiative transfer model. The resulting synthetic observations can be directly compared to real data to discriminate between theoretical models of star formation. We find that the most promising tracers of collapse mechanism are the ratio between blue and red peaks in the CS $J=2-1$ line profile, and the intensity ratios of lines from molecules affected differently by freeze-out on to grains, such as $\mathrm{N}_{2} \mathrm{H}^{+}$and CS/HCN. Specifically, CS blue/red ratios of $<3(>5), \mathrm{N}_{2} \mathrm{H}^{+} / \mathrm{CS}$ ratios of $>0.6(<0.2)$, and $\mathrm{HCN} / \mathrm{N}_{2} \mathrm{H}^{+}$ratios of $<1(>1)$ are indicative of subcritical (supercritical) collapse. These criteria, and the good match with model line profile shapes, suggest that L1498 resulted from subcritical initial conditions, despite being marginally supercritical at present.

\section{ACKNOWLEDGEMENTS}

FDP is funded by the Science and Technology Facilities Council. Some figures in this paper were made using SPLASH (Price 2007).

\section{DATA AVAILABILITY}

The data underlying this article will be shared on reasonable request to the corresponding author.

\section{REFERENCES}

Banerji M., Viti S., Williams D. A., Rawlings J. M. C., 2009, ApJ, 692, 283 Bate M. R., Tricco T. S., Price D. J., 2014, MNRAS, 437, 77

Beltrán M. T. et al., 2019, A\&A, 630, A54

Brinch C., Hogerheijde M. R., 2010, A\&A, 523, A25

Coutens A., Commerçon B., Wakelam V., 2020, A\&A, 643, A108

Crutcher R. M., 2012, ARA\&A, 50, 29

Crutcher R. M., Hakobian N., Troland T. H., 2009, ApJ, 692, 844

Fiedler R. A., Mouschovias T. C., 1993, ApJ, 415, 680

Holdship J., Viti S., Jiménez-Serra I., Makrymallis A., Priestley F., 2017, AJ, 154,38

Jiang H., Li H.-b., Fan X., 2020, ApJ, 890, 153

Kirk J. M., Ward-Thompson D., Crutcher R. M., 2006, MNRAS, 369, 1445

Lee H.-H., Roueff E., Pineau des Forets G., Shalabiea O. M., Terzieva R., Herbst E., 1998, A\&A, 334, 1047 
Lin S.-J., Pagani L., Lai S.-P., Lefèvre C., Lique F., 2020, A\&A, 635, A188 Lippok N. et al., 2013, A\&A, 560, A41

McElroy D., Walsh C., Markwick A. J., Cordiner M. A., Smith K., Millar T. J., 2013, A\&A, 550, A36

Machida M. N., Higuchi K., Okuzumi S., 2018, MNRAS, 473, 3080

Mouschovias T. C., 1976, ApJ, 206, 753

Öberg K. I., Bergin E. A., 2020, Phys. Rep., 893, 1

Pagani L., Lesaffre P., Jorfi M., Honvault P., González-Lezana T., Faure A., 2013, A\&A, 551, A38

Price D. J., 2007, Publ. Astron. Soc. Aust., 24, 159

Price D. J. et al., 2018, Publ. Astron. Soc. Aust., 35, e031

Priestley F. D., Viti S., Williams D. A., 2018, AJ, 156, 51

Priestley F. D., Wurster J., Viti S., 2019, MNRAS, 488, 2357

Schöier F. L., van der Tak F. F. S., van Dishoeck E. F., Black J. H., 2005, A\&A, 432, 369

Soam A. et al., 2018, ApJ , 861, 65

Soam A. et al., 2019, ApJ, 883, 95
Tafalla M., Myers P. C., Caselli P., Walmsley C. M., Comito C., 2002, ApJ, 569,815

Tafalla M., Santiago-García J., Myers P. C., Caselli P., Walmsley C. M., Crapsi A., 2006, A\&A, 455, 577

Tassis K., Mouschovias T. C., 2004, ApJ, 616, 283

Tassis K., Willacy K., Yorke H. W., Turner N. J., 2012a, ApJ, 753, 29

Tassis K., Willacy K., Yorke H. W., Turner N. J., 2012b, ApJ, 754, 6

Tassis K., Willacy K., Yorke H. W., Turner N. J., 2014, MNRAS, 445, L56

Tritsis A., Panopoulou G. V., Mouschovias T. C., Tassis K., Pavlidou V., 2015, MNRAS, 451, 4384

Wurster J., 2016, Publ. Astron. Soc. Aust., 33, e041

Wurster J., Bate M. R., Price D. J., 2018, MNRAS, 475, 1859

This paper has been typeset from a $\mathrm{T}_{\mathrm{E}} \mathrm{X} / \mathrm{LT} \mathrm{E} \mathrm{X}$ file prepared by the author. 